\title{
Apports de lignines et alimentation du lapin en croissance. I. Conséquences sur la digestion et le transit
}

\author{
T Gidenne, JM Perez \\ avec la collaboration technique d'A Lapanouse et M Ségura
}

INRA, station de recherches cunicoles, BP 27, F 31326 Castanet-Tolosan cedex, France

(Reçu le 13 septembre 1993; accepté le 10 janvier 1994)

\begin{abstract}
Résumé - L'effet de la teneur et de la nature des lignines sur la digestion et le transit du lapin en croissance (31 à 73 j d'âge) a été étudié à l'aide de 5 aliments : un aliment de référence à faible teneur en lignine $A D L(R 0)$, et des aliments à taux croissants de lignines provenant pour un tiers soit de luzerne déshydratée (régimes L1 et L2) soit de tourteau de pépins de raisin (régimes $\mathrm{P} 1$ et $\mathrm{P} 2$ ). La digestibilité fécale de la matière organique décroît linéairement avec le taux de lignines ( -2 points par point d'ADL, $r^{2}=0,98$ ) : la digestibilité des fibres est abaissée alors que celle des protéines n'est pas affectée. Le temps de séjour moyen total $(20,4 \mathrm{~h})$ décroît $\left(-20 \%\right.$ entre Ro et P2) avec la teneur en ADL $\left(r^{2}=\right.$ 0,93 ). Le temps de séjour des particules grossières (non recyclées par la cæcotrophie) dans l'ensemble cæcum-côlon ( $\mathrm{TCgp}=11,0 \mathrm{~h}$ en moyenne) est 2 fois plus court que celui des petites particules (TCpp = $21,7 \mathrm{~h}$ en moyenne) ; seul ce dernier est significativement réduit avec la teneur en ADL ( $-33 \%$ entre R0 et L2).
\end{abstract}

\section{lapin / digestion / transit / lignines / fibres alimentaires}

Summary - Dietary lignin in growing rabbits. I. Consequences on digestibility and rate of passage. The effect of the origin and level of dietary lignin on the digestibility and rate of passage of growing rabbits ( $31-73 d$ of age) was studied using 5 diets (table 1): a reference diet $(R 0)$ with a low acid detergent lignin ( $A D L$ ) content (2\%); and 4 diets with increasing $A D L$ content (table III) from 2 main $A D L$ origins, lucerne $(L 1, L 2)$ or grape-pip meal $(P 1, P 2)$. Organic matter digestibility decreased linearly according to the level of $A D L\left(-2\right.$ points per point of $\left.A D L, \mathrm{r}^{2}=0.98\right)$. Increasing the $A D L$ level mainly affected the fibre degradation, without incidence on protein digestibility. The mean retention time in the whole tract decreased $(-20 \%$ between $R O$ and $P 2)$ as dietary $A D L$ increased $\left(\mathrm{r}^{2}=0.93\right)$. The rate of passage of raw particles (unrecyled by caecotrophy) in the caeco-colic compartment was 2-fold shorter ( $T C_{G D}$ $=11.0 \mathrm{~h})$ than that of the fine particles $\left(T C_{p p}=21.7 \mathrm{~h}\right)$. Only the former was significantly reduced according to the dietary $A D L$ level ( $-33 \%$ between $R 0$ and $L 2)$.

rabbit / digestion / rate of passage / lignin / dietary fibre 


\section{INTRODUCTION}

Les lignines des parois végétales sont relativement bien identifiées au plan biochimique (Monties, 1980), mais leur analyse dans les aliments et les digesta des animaux demeure très imprécise (Giger, 1985). Chez les ruminants, les effets nutritionnels négatifs des lignines des fourrages ont été clarifiés : effet de barrière physique à l'attaque microbienne des fibres, effet d'inhibition des fermentations (Jung et Fahey, 1983). En revanche chez les monogastriques, ces effets sont plus difficiles à mettre en évidence. Ainsi, réputées indigestibles, les lignines présentent des digestibilités fécales fréquemment positives, voire fortes (10 à $50 \%$ ), que ce soit chez le porc (Perez, 1991) ou chez le lapin (Falcao E Cunha, 1988). Chez ce dernier, des effets favorables des lignines ont même été observés concernant l'activité fermentaire cæcale (Gidenne, 1986) ou la dégradation des fibres (Falcao E Cunha et Lebas, 1986). Au-delà des problèmes analytiques, la nature comme la teneur en lignines des aliments peuvent être à l'origine de ces effets. Ainsi, les aliments destinés aux lapins contiennent souvent des sous-produits agro-industriels (Pérez De Ayala, 1989 ; Fraga et al, 1991 ; Merino et Carabano, 1992), dont certains sont très lignifiés, mais également riches en tannins et cutines (marc de raisin, pellicules de colza...), qui contaminent le résidu lignine ADL (acid detergent lignin).

L'effet des lignines sur la digestion a fait l'objet de quelques travaux chez le lapin, principalement à l'aide de régimes alimentaires simplifiés (Lenz et Schurch, 1967 ; Parigi-Bini et Chiericato, 1980 ; Gidenne, $1987 a, b)$. C'est pourquoi notre travail s'appuie sur des modèles alimentaires plus complexes, relativement proches de ceux utilisés en élevage rationnel, afin d'étudier les effets de la nature et de la teneur en lignines sur la digestion et le transit du lapin en croissance. La seconde partie de ce tra- vail (Perez et al, 1994) concernera l'influence des lignines sur les performances de croissance et sur la mortalité enregistrées dans un réseau de 5 stations expérimentales cunicoles.

\section{MATÉRIEL ET MÉTHODES}

\section{Aliments et animaux}

L'objectif de la formulation était d'obtenir une variation du taux et de la nature des lignines $(A D L)$, sans variations des autres fractions pariétales (hémicelluloses et cellulose Van Soest) ni des apports protéiques. Au total, 5 aliments ont été fabriqués à partir de matières premières courantes (tableau I) : un aliment de référence à faible teneur en lignine $A D L$ (régime Ro), et des aliments à taux croissants d'ADL provenant pour un tiers (tableau II) soit de luzerne déshydratée (régimes L1 et L2), soit de tourteau de pépins de raisin (régimes $\mathrm{P} 1$ et $\mathrm{P} 2$ ). D'autre part, ces aliments contiennent une base amylacée (blé) et protéique (tourteau de soja) commune. Chaque aliment a été distribué ad libitum à 15 lapereaux mâles Néo-Zélandais Blancs, logés en cage individuelle à métabolisme, depuis le sevrage ( $31 \mathrm{j}$ d'âge) jusqu'à l'abattage (73 j d'âge). Les poids vifs et la consommation ont été mesurés individuellement chaque semaine.

\section{Mesures de digestibilité et de transit}

Les mesures de digestibilité apparente fécale ont été réalisées entre 6 et 8 sem d'âge pendant 2 périodes de $4 \mathbf{j}$ (Colin et Lebas, 1976) sur un effectif initial de 9 lapereaux par régime, sachant que seuls les animaux n'ayant pas présenté d'anomalie d'ingestion ou d'excrétion sont retenus pour l'analyse statistique.

Parallèlement, les mesures de transit digestif ont été réalisées pour chaque régime sur 6 lapereaux âgés de 7 sem. La cinétique d'excrétion fécale d'une dose de particules fibreuses marquées au ${ }^{141}$ cérium $(\approx 2 \mathrm{kBq} /$ dose) a été suivie pendant $3 \mathrm{j}$, selon la méthode décrite par Gidenne (1993). Le temps de séjour moyen (TSM) des aliments est calculé selon la formule générale pro- 
Tableau I. Composition centésimale des aliments expérimentaux.

\begin{tabular}{lcrrrc}
\hline Régimes & $L 2$ & $L 1$ & $R 0$ & $P 1$ & $P 2$ \\
\hline Blé & & & & & \\
Son de blé & 35,0 & 37,0 & 38,9 & 35,3 & 31,6 \\
Luzerne déshydratée & - & 2,0 & 4,0 & 2,0 & - \\
Paille de blé & - & 15,6 & 7,8 & 4,0 & - \\
Pulpes de betteraves & 4,0 & 1,5 & 3,0 & 5,75 & 8,5 \\
Coques de tournesol & 11,8 & 7,0 & 10,0 & 10,5 & 11,0 \\
Coques de soja & - & 5,9 & - & 6,0 & 12,0 \\
Tourteau tournesol & 12,0 & 7,5 & 15,0 & 7,5 & - \\
Tourteau soja 48 & 8,0 & 11,7 & - & 7,5 & 15,0 \\
Tourteau pépins raisin & - & - & - & 13,4 & 11,0 \\
Minéraux et vitamines & 5,9 & 5,7 & 5,9 & 2,5 & 5,0 \\
& & & & 5,75 & 5,9 \\
\hline
\end{tabular}

Tableau II. Proportions (\%) selon les régimes de la fraction ADL apportée par les principales matières premières.

\begin{tabular}{lccccc}
\hline Régimes & $L 2$ & $L 1$ & $R 0$ & $P 1$ & $P 2$ \\
\hline Luzerne déshydratée & 32,2 & 32,9 & 34,7 & 6,4 & - \\
Paille de blé & - & 3,7 & 16,1 & 11,4 & 10,2 \\
Coques de tournesol & 43,0 & 33,2 & - & 26,1 & 32,0 \\
Tourteau tournesol & 17,0 & 13,2 & - & 12,6 & 15,6 \\
Tourteau pépins raisin & - & - & - & 29,2 & 35,7 \\
\hline
\end{tabular}

posée par Faichney (1975): TSM $=\sum M_{i} T_{i} / \Sigma$ $M_{i}$, où $T_{i}$ représente le temps moyen écoulé entre To (administration du marqueur) et la je récolte, et $M_{i}$ la masse de marqueur excrété entre $T_{j-1}$ et $T_{i}$

Le temps de transit (TT) est le délai (temps moyen) de première apparition du marqueur dans les fèces, qui est assimilable dans le cas du lapin au temps de passage dans l'intestin grêle et le côlon distal (Gidenne et al, 1991).

En outre, nous avons remarqué que la cæcotrophie a pour effet d'infléchir la décroissance de la concentration ( $\mathrm{Ct}$ ) fécale en marqueur (fig 1), du fait d'un recyclage de particules marquées. Nous avons donc réalisé un double ajustement de la partie décroissante de la cinétique avant et après la cæcotrophie, par régression non linéaire selon une loi exponentielle $\left(\mathrm{Ct}=\mathrm{Co}^{\circ} \exp ^{-\mathrm{kt}}\right)$. Nous avons défini 2 index TCgp et TCpp comme l'inverse des constantes de temps des 2 équations, représen- tant chacun un temps de séjour dans le compartiment "cæcum-côlon proximal». En accord avec les travaux antérieurs (Björnhag, 1972 ; Jilge, 1982), TCgp correspondrait au temps de séjour des particules marquées grossières $(>0,3 \mathrm{~mm}$ ) excrétées rapidement dans les fèces dures. TCpp correspondrait aux particules marquées plus fines, refoulées par le côlon proximal en période de formation de crottes dures, et qui sont donc susceptibles d'être incorporées dans les cæcotrophes et réingérées.

\section{Analyses chimiques}

Les analyses suivantes ont été réalisées sur les aliments et les fèces : matière sèche (MS) par dessiccation durant $24 \mathrm{~h}$ à $103^{\circ} \mathrm{C}$, cendres par calcination durant $5 \mathrm{~h}$ à $550^{\circ} \mathrm{C}$, matières azotées 


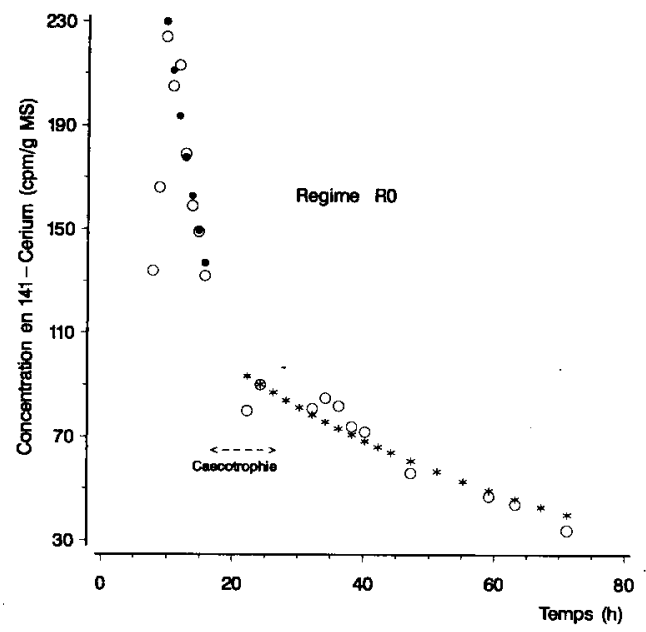

Fig 1. Exemple d'analyse de la cinétique de concentration fécale en marqueur, en fonction de la cæcotrophie. o : valeurs observées ; $\bullet$ : valeurs obtenues par ajustement non linéaire, avant la première phase de cæcotrophie $(C=$ Co•ex-kt, $1 / \mathrm{k}=$ TCgp) ; ${ }^{*}$ : valeurs obtenues par ajustement non linéaire, après la première phase de cæcotrophie $\left(C=C o \cdot e x^{-k t}, 1 / k=T C p p\right)$.

totales (méthode Kjeldhal, $\mathrm{N} \times 6,25$ ), énergie brute (calorimètre adiabatique PARR) ; les constituants pariétaux (NDF, ADF, et lignine $A D L$ ) ont été analysés séquentiellement selon la méthode de Van Soest et al (1991), après un prétraitement avec une amylase thermostable (Termamyl 120L, Novo Nordisk, Fontenay, France). La lignine permanganate des aliments $\left(\mathrm{KMnO}_{4}\right)$ a été dosée selon la technique de $V$ an Soest et Wine (1968), après extraction avec les détergents neutres et acides. La teneur en constituants cellulaires non azotés (CCNA) a été estimée par différence selon la relation :

$$
\operatorname{CCNA}(\%)=\text { MO }(\%)-\text { MAT }(\%)-\operatorname{NDF}(\%)
$$

\section{Analyses statistiques}

Le traitement statistique des données a été réalisé par analyse de variance à un facteur de clas- sification (effet régime) selon la procédure GLM du logiciel SAS (SAS, 1988). La comparaison multiple des moyennes a été effectuées à l'aide du test de Bonferroni. Les ajustements de la phase décroissante des courbes de concentration en marqueur ont été calculés par régression nonlinéaire (procédure NLIN, SAS 1988).

\section{RÉSULTATS}

D'après les résultats d'analyses, répétées dans plusieurs laboratoires (tableau III), les aliments diffèrent bien essentiellement par leurs teneurs en lignines (sulfurique ou permanganate), les fractions cellulose (ADF$A D L$ ) et hémicelluloses (NDF-ADF) étant similaires entre les régimes (tableau III). Les teneurs en lignine des aliments Ro, $L 1$, L2 ne diffèrent pas selon la méthode de dosage, en revanche la teneur en lignine ADL est sensiblement supérieure à la teneur en lignine permanganate pour les aliments $P 1$ et $P 2$. Par ailleurs, les aliments sont isoazotés et présentent de faibles variations de teneurs en acides aminés et en amidon. Parallèlement à la hausse de la teneur en fibres, la granulométrie devient plus grossière (tableau IV). Ainsi, pour les aliments contenant de la paille (R0, P1, P2), la proportion de particules supérieures à $0,3 \mathrm{~mm}$ (non recyclée dans les cæcotrophes) passe de $44,6 \%$ (R0) à 53,5\% (P2).

La croissance des animaux du sevrage à l'âge d'abattage est similaire entre les traitements. La consommation d'aliment augmente $(P=0,13)$ avec les apports de fibres (tableau $\mathrm{V}$ ) et l'indice de consommation s'accroît significativement avec la teneur en $A D L:+18 \%$ entre R0 et P2. L'ingéré d'énergie digestible ne varie pas significativement selon les aliments, de sorte que l'indice de conversion énergétique ne varie pas en fonction des régimes (écart maximum inférieur à 5\%).

La digestibilité apparente fécale "CUDa" (tableau VI) de la matière organique (comme le CUDa de l'énergie) diminue linéairement 
Tableau III . Composition chimique moyenne des aliments expérimentaux (\% brut).

\begin{tabular}{|c|c|c|c|c|c|c|}
\hline Régimes & $n$ & L2 & $L 1$ & Ro & $P 1$ & $P 2$ \\
\hline Matière sèche & 6 & 90,1 & 89,6 & 89,5 & 89,7 & 89,7 \\
\hline Cendres & 5 & 9,2 & 8,7 & 8,6 & 8,8 & 8,7 \\
\hline Matières grasses & 3 & 1,9 & 1,9 & 2,1 & 1,9 & 2,2 \\
\hline $\operatorname{MAT}(\mathrm{N} \times 6,25)$ & 6 & $16,5 \pm 0,3 b$ & $17,0 \pm 0,5$ & $17,0 \pm 0,3$ & $16,4 \pm 0,6$ & $16,6 \pm 0,5$ \\
\hline Lysine & 1 & 0,74 & 0,77 & 0,81 & 0,75 & 0,73 \\
\hline AA soufrés & 1 & 0,61 & 0,64 & 0,68 & 0,63 & 0,60 \\
\hline Thréonine & 1 & 0,60 & 0,61 & 0,62 & 0,60 & 0,60 \\
\hline Amidon & 4 & 23,0 & 23,8 & 26,2 & 23,6 & 21,2 \\
\hline CCNA a & 5 & 33,9 & 35,1 & 38,3 & 34,2 & 30,0 \\
\hline Cellulose brute & 5 & $17,2 \pm 1,1$ & $14,5 \pm 0,3$ & $12,0 \pm 0,4$ & $15,6 \pm 0,4$ & $19,1 \pm 0,6$ \\
\hline NDF & 6 & $30,4 \pm 1,6$ & $28,7 \pm 1,7$ & $25,6 \pm 1,8$ & $30,1 \pm 2,3$ & $33,2 \pm 1,0$ \\
\hline ADF & 6 & $19,5 \pm 0,8$ & $17,2 \pm 0,5$ & $14,2 \pm 0,7$ & $18,2 \pm 0,9$ & $22,0 \pm 0,9$ \\
\hline Hémicellulose (NDF-ADF) & & 10,9 & 11,5 & 11,4 & 11,9 & 11,2 \\
\hline Cellulose (ADF-ADL) & & 13,6 & 13,2 & 12,2 & 13,5 & 14,4 \\
\hline \multicolumn{7}{|l|}{ Lignines } \\
\hline $\mathrm{KMnO}_{4}$ & 1 & 5,9 & 3,8 & 2,2 & 4,1 & 4,9 \\
\hline ADL & 5 & $5,9 \pm 0,5$ & $4,0 \pm 0,4$ & $2,0 \pm 0,4$ & $4,7 \pm 0,5$ & $7,6 \pm 0,5$ \\
\hline
\end{tabular}

$n:$ nombre de sites d'analyse. a CCNA = MO - MAT - NDF; ${ }^{\mathrm{a}} \pm$ écart type

Tableau IV . Analyse granulométrique des aliments expérimentaux.

\begin{tabular}{lrrrrr}
\hline Classes de particules "t» (\%) & $L 2$ & $L 1$ & $R 0$ & $P 1$ & $P 2$ \\
\hline $\mathrm{t}>1 \mathrm{~mm}$ & 14,4 & 9,6 & 11,3 & 14,9 & 16,3 \\
$1 \mathrm{~mm}>\mathrm{t}>0,5 \mathrm{~mm}$ & 21,0 & 20,2 & 21,4 & 22,2 & 22,4 \\
$0,5>\mathrm{t}>0,315$ & 12,9 & 14,1 & 11,9 & 14,1 & 14,8 \\
$0,315>\mathrm{t}>0,1$ & 13,4 & 14,0 & 13,3 & 12,6 & 12,1 \\
$0,1>\mathrm{t}>0,05$ & 4,1 & 3,6 & 3,6 & 3,4 & 3,5 \\
$\mathrm{t}<0,05 \mathrm{~mm}$ & 34,2 & 38,6 & 38,5 & 32,7 & 30,8 \\
\hline
\end{tabular}


Tableau V . Performances de croissance entre 31 et 73 j d'âge.

\begin{tabular}{|c|c|c|c|c|c|c|c|}
\hline $\begin{array}{l}\text { Régimes } \\
\text { Lignine } A D L(\%)\end{array}$ & $\begin{array}{r}L 2 \\
5,9\end{array}$ & $\begin{array}{l}L 1 \\
4,0\end{array}$ & $\begin{array}{l}R 0 \\
2,0\end{array}$ & $\begin{array}{l}P 1 \\
4,7\end{array}$ & $\begin{array}{l}P 2 \\
7,6\end{array}$ & ETR & $\begin{array}{l}\text { Sign } \\
\text { Stat }\end{array}$ \\
\hline Poids à 31 j (g) & 679 & 690 & 678 & 705 & 684 & 93 & NS \\
\hline Gain de poids ( $g / j)$ & 34,5 & 36,4 & 35,8 & 35,4 & 33,9 & 3,7 & NS \\
\hline $\begin{array}{l}\text { Consommation } \\
(\mathrm{g} / \mathrm{j}) \\
(\mathrm{MJ} E \mathrm{ED} / \mathrm{j})\end{array}$ & $\begin{array}{r}104,0 \\
1,06\end{array}$ & $\begin{array}{r}104,6 \\
1,12\end{array}$ & $\begin{array}{c}97,2 \\
1,07\end{array}$ & $\begin{array}{l}105,6 \\
1,06\end{array}$ & $\begin{array}{c}108,3 \\
1,00\end{array}$ & $\begin{array}{c}12,0 \\
0,12\end{array}$ & $\begin{array}{l}\text { NS } \\
\text { NS }\end{array}$ \\
\hline $\begin{array}{l}\text { Indice de consomm } \\
\text { (kg/kg gain) } \\
\text { (MJ ED/kg gain ) }\end{array}$ & $3,02^{b}$ & $\begin{array}{l}2,90 \mathrm{bc} \\
31,0\end{array}$ & $\begin{array}{l}2,72^{c} \\
29,8\end{array}$ & $\begin{array}{l}3,00^{b c} \\
29,7\end{array}$ & $\begin{array}{l}3,21^{a} \\
29,7\end{array}$ & $\begin{array}{l}0,20 \\
2,1\end{array}$ & NS \\
\hline
\end{tabular}

NS : $P>0,10 ;{ }^{*}: P<0,05 ;{ }^{* *}: P<0,01, a, b, c:$ les moyennes ayant une lettre en commun ne diffèrent pas au seuil $P<0,05$. ETR : écart type résiduel.

Tableau VI. Digestibilité apparente fécale (\%) et valeur nutritive des aliments expérimentaux.

\begin{tabular}{|c|c|c|c|c|c|c|c|}
\hline $\begin{array}{l}\text { Régimes } \\
\text { Lignine ADL (\%) }\end{array}$ & $\begin{array}{c}L 2 \\
5,9 \\
(n=7)\end{array}$ & $\begin{array}{c}L 1 \\
4,0 \\
(n=8)\end{array}$ & $\begin{array}{c}R 0 \\
2,0 \\
(n=7)\end{array}$ & $\begin{array}{r}P 1 \\
4,7 \\
(n=9)\end{array}$ & $\begin{array}{c}P 2 \\
7,6 \\
(n=7)\end{array}$ & ETR & $\begin{array}{l}\text { Sign } \\
\text { Stat }\end{array}$ \\
\hline Matière organique & $66,1^{10}$ & $70,2^{b}$ & $73,8^{a}$ & $67,0^{c}$ & $61,2^{d}$ & 2,04 & ** \\
\hline Énergie & $65,6^{c}$ & $69,2^{b}$ & $73,1^{a}$ & $65,3^{c}$ & $59,6^{d}$ & 2,03 & $\star \star *$ \\
\hline MAT & 82,6 & 81,7 & 80,8 & 79,4 & 81,3 & 2,57 & NS \\
\hline $\mathrm{CCNA}^{1}$ & $89,1^{b}$ & $91,4^{a b}$ & $92,6^{a}$ & $92,1^{\mathrm{a}}$ & $92,2^{\mathrm{a}}$ & 1,65 & $\star \star$ \\
\hline NDF & $34,8^{\mathrm{bc}}$ & $39,3^{a b}$ & $44,0^{\mathrm{a}}$ & $35,7^{b c}$ & $32,3^{c}$ & 3,76 & ** \\
\hline ADF & $21,5^{a b}$ & $23,7^{a b}$ & $24,2^{\mathrm{a}}$ & $18,9^{a b}$ & $17,6^{b}$ & 4,43 & * \\
\hline ADL & $22,2^{a}$ & $24,5^{a}$ & $20,8^{a}$ & $7,7^{b}$ & $10,1^{b}$ & 3,15 & $\star \star$ \\
\hline Hémicelluloses ${ }^{2}$ & $54,7^{b}$ & $58,8^{b}$ & $63,6^{a}$ & $56,9^{b}$ & $53,1^{b}$ & 3,56 & $\star \star$ \\
\hline Cellulose $^{3}$ & 21,1 & 23,4 & 24,8 & 23,1 & 21,8 & 5,64 & NS \\
\hline ED (MJ/kg brut) & 10,15 & 10,68 & 10,98 & 10,01 & 9,27 & & \\
\hline MAD (g/kg brut) & 136 & 139 & 137 & 130 & 135 & & \\
\hline MAD/ED (g/MJ) & 13,4 & 13,0 & 12,5 & 13,0 & 14,6 & & \\
\hline
\end{tabular}

$n$ : nombre de lapereaux retenus pour le calcul de digestibilité. $\quad{ }^{1}$ CCNA $=$ MO - MAT - NDF $;{ }^{2}$ résidu NDF-ADF ; 3 résidu ADF-ADL. NS, *, a b voir tableau $V$. 
d'environ 2 points par point supplémentaire d'ADL :

$$
\text { CUDa } M O(\%)=78,3-2,19 \text { ADL }(\%)
$$

$r^{2}=0,98 \quad$ ETR $=0,85 \quad C V=1,3 \%$

La relation est moins étroite avec le critère lignine permanganate $\left(r^{2}=0,62 ; \mathrm{CV}=\right.$ $4,9 \%$ ). La digestibilité de l'azote est relativement élevée ( $80 \%$ en moyenne), et n'est pas affectée par la teneur en lignines des rations. La fraction CCNA (contenant en moyenne $69 \%$ d'amidon) présente une digestibilité très élevée $(91,5 \%$ en moyenne), mais négativement affectée par l'addition de lignines provenant de luzerne. La digestibilité de la fraction NDF décroît linéairement avec le taux d'ADL $\left(r^{2}=0,94\right)$, principalement du fait d'une réduction $(-16,5 \%$ entre R0 et $\mathrm{P} 2)$ de la dégradation de la fraction hémicelluloses (NDF-ADF), et sans effet apparent de la nature des lignines ( $L$ ou $P$ ). Le CUDa de la fraction cellulose (ADF-ADL) ne dépasse pas $25 \%$ et ne varie pas significativement en fonction des aliments. Ainsi, le taux de NDF indigestible demeure constant entre les régimes; et le taux d'ADF indigestible varie au maximum de 3,4 à $4,2 \%$ entre Ro et $L 2$.
Le temps de séjour moyen total (tableau VIi) des aliments décroît (-20\% entre les régimes extrêmes) avec la teneur en $A D L$ des aliments $\left(r^{2}=0,93\right)$. Il est également associé à une élévation du niveau moyen d'ingestion $\left(r^{2}=0,97\right)$, mais la corrélation, sur les données individuelles $(n=27)$, entre la consommation d'aliment et le TSM demeure faible $\left(r^{2}=0,25\right)$. II faut aussi noter que la corrélation individuelle entre la digestibilité de la matière sèche et le TSM est hautement significative $\left(P<0,001 ; r^{2}=\right.$ $0,48 ; n=27)$. Une réduction significative du temps de transit «TT» est également observée, en particulier entre les régimes $\mathrm{R} 0$ et $\mathrm{P} 2$. Aucune variation significative de l'index TCgp n'est détectable du fait de fortes variations inter-individuelles; en revanche, l'index TCpp (basé sur environ 30 à $40 \%$ de la quantité de marqueur excrété) est significativement réduit avec la teneur en ADL des aliments.

\section{DISCUSSION}

Le résidu $A D L$, isolé après action de l'acide sulfurique sur le résidu $A D F$, comprend, en

Tableau VII. Mesures de transit digestif.

\begin{tabular}{|c|c|c|c|c|c|c|c|}
\hline $\begin{array}{l}\text { Régimes } \\
\text { Lignine } A D L(\%)\end{array}$ & $\begin{array}{c}L 2 \\
5,9 \\
(n=6)\end{array}$ & $\begin{array}{c}L 1 \\
4,0 \\
(n=5)\end{array}$ & $\begin{array}{c}R O \\
2,0 \\
(n=5)\end{array}$ & $\begin{array}{c}P 1 \\
4,7 \\
(n=5)\end{array}$ & $\begin{array}{c}P 2 \\
7,6 \\
(n=6)\end{array}$ & ETR & $\begin{array}{l}\text { Sign } \\
\text { Stat }\end{array}$ \\
\hline Ingéré $(\mathrm{gMS} / \mathrm{j})^{1}$ & $101,9^{b}$ & $94,6^{\mathrm{ab}}$ & $87,6^{\mathrm{a}}$ & $95,8^{a b}$ & $105,2^{b}$ & 6,0 & * \\
\hline $\begin{array}{l}\text { TSM (h) } \\
\text { TT (h) } \\
\text { TCgp (h) } \\
\text { TCpp (h) }\end{array}$ & $\begin{array}{c}18,6^{\mathrm{b}} \\
6,6^{\mathrm{a}} \\
9,3 \\
17,7^{\mathrm{b}}\end{array}$ & $\begin{array}{c}21,6^{\mathrm{ab}} \\
5,1^{\mathrm{ab}} \\
13,1 \\
26,7^{\mathrm{a}}\end{array}$ & $\begin{array}{c}23,0^{\mathrm{a}} \\
7,0^{\mathrm{a}} \\
12,1 \\
26,6^{\mathrm{a}}\end{array}$ & $\begin{array}{c}20,6^{\mathrm{ab}} \\
5,7^{\mathrm{ab}} \\
11,0 \\
20,9^{\mathrm{ab}}\end{array}$ & $\begin{array}{c}18,4^{\mathrm{b}} \\
4,2^{\mathrm{b}} \\
10,1 \\
19,7^{\mathrm{ab}}\end{array}$ & $\begin{array}{l}1,81 \\
1,11 \\
3,27 \\
2,96\end{array}$ & $\begin{array}{r}\star * \\
\text { NS } \\
\star \star\end{array}$ \\
\hline
\end{tabular}

$\mathrm{n}$ : Nombre de lapereaux retenus. ${ }^{1}$ Consommation d'aliment durant les mesures de transit digestif.TSM : temps de séjour moyen $(\Sigma \mathrm{Mi} \cdot \mathrm{Ti} / \Sigma \mathrm{Mi}) . \Pi \mathrm{T}$ : temps de transit ou temps de première apparition du marqueur dans les fèces. TCgp : temps de séjour moyen des particules grossières, non recyclées par la cacotrophie. TCpp : temps de séjour moyen des fines particules, potentiellement recyclées par la cæcotrophie. NS, ", a,b : voir tableau V. 
sus des lignines, des cutines mais aussi des produits de condensation tannins-protéines et des protéines dénaturées à la chaleur. Sa valeur est en général supérieure à celle de la lignine permanganate sauf dans le cas d'échantillons riches en tannins (Giger, 1985), ou d'échantillons riches en cutines. Ces dernières résistent à l'acide sulfurique et au permanganate (Van Soest et al, 1991). Elles sont donc présentes dans le résidu ADL, mais ne sont pas comptées dans la lignine permanganate. Ainsi, dans le cas des régimes $P 1$ et surtout $P 2$ contenant du tourteau de pépins de raisin riche en tannins et cutines, les écarts de teneurs entre ADL et lignine permanganate proviendraient donc surtout d'une surestimation du résidu ADL.

La réduction du CUD de la MO, plus que proportionnelle à l'élévation du taux d'ADL, indique un effet négatif propre des lignines sur la digestion des aliments, au-delà d'un effet de lest, sans que nous ayons constaté un effet de la nature des lignines ( $L$ ou $P$ ). En revanche, des études antérieures ont montré un effet négatif peu marqué des lignines, et variable selon le sous-produit utilisé comme source principale de lignines: réduction peu importante de la digestibilité avec le marc de raisin (Motta, 1990 ; ParigiBini et Chiericato, 1980 ; Falcao e Cunha et Lebas, 1986) ou les pellicules de colza (Gidenne, 1987b), voire quasi nulle lors de l'utilisation de peaux de tomates (Falcao e Cunha et Lebas, 1986).

Une réduction de la digestibilité des protéines est généralement observée lors de l'élévation de la teneur en lignines alimentaires (Motta, 1990 ; Falcao e Cunha, 1988; Gidenne, 1987b). Mais lors de ces expérimentations, il est difficile de distinguer l'effet propre des lignines de celui provenant de la nature des protéines apportées par les matières premières. Nos modèles alimentaires formulés sur la base d'un apport constant en protéines digestibles permettent de mettre en évidence un effet direct des lignines. Dans ces conditions, nos résultats n'indiquent pas d'effet négatif des lignines per se sur la dégradation des protéines, bien que l'ingestion de lignines quadruple entre R0 et P2.

La dégradation des constituants pariétaux est relativement élevée, dépassant $60 \%$ pour la fraction hémicelluloses du régime $R O$ (coques de soja et pulpes de betteraves). De même, Falcao e Cunha (1988) enregistre une digestibilité des hémicelluloses supérieure à $65 \%$ avec des régimes à base de coques de soja. La dégradation des fibres est négativement liée à la teneur en $A D L$, sans incidence de la nature des lignines; avec la lignine permanganate, cette liaison est moins étroite. En revanche, la nature des lignines ( $L$ ou $P)$ semble avoir un effet sur la dégradation de l'ensemble des constituants cellulaires non azotés (CCNA).

L'originalité des mesures de transit digestif réside dans le fait qu'elles ont été réalisées sur des animaux jeunes (7 sem) et de même âge que ceux utilisés pour la mesure de digestibilité. En outre, à notre connaissance, l'incidence de la nature et de la teneur en lignines alimentaires sur le transit n'a pas fait l'objet d'études spécifiques. Nous observons ainsi un transit total (TSM) plus rapide avec un taux élevé de lignines, sans relation étroite avec la consommation d'indigestible pariétal (NDF ou ADF). L'origine botanique de la fraction lignines des aliments semble avoir une influence sur le transit digestif. Ainsi, lors d'un apport de lignines par la paille et le tourteau de pépins de raisin (aliments $P 1$ et $P 2$ ), la diminution du TSM proviendrait surtout d'une diminution du temps de séjour dans les parties tubulaires du tractus digestif (principalement intestin grêle) ; cependant, on ne peut exclure un effet associé de la granulométrie relativement plus grossière pour ces régimes. En revanche, la diminution du TSM dans le cas des régimes à base de luzerne résulterait plutôt d'une réduction du temps 
de séjour dans l'ensemble cæcum-côlon puisque on ne constate aucune différence de temps de transit (TT).

Les valeurs des index TCgp et TCpp suggèrent que le temps de séjour des particules marquées grossières (non recyclées par la cæcotrophie) dans l'ensemble cæcum-côlon proximal (TCgp $=11,0 \mathrm{~h}$ en moyenne) est 2 fois plus court que celui des petites particules (TCpp $=21,7 \mathrm{~h}$ en moyenne). Des valeurs similaires de rétention de fines particules $(<0,075 \mathrm{~mm})$ et de grosses particules ( $>0,3 \mathrm{~mm}$ ) ont été observées antérieurement chez des lapins adultes nourris ad libitum (Uden et Van Soest, 1982). En revanche chez l'adulte rationné (Gidenne, 1993), les valeurs de temps de séjour sont plus élevées ( 26 à 35 h), et les écarts entre particules marquées fines et grossières sont plus faibles ( 7 à $10 \mathrm{~h}$ ). L'élévation du taux de lignines entraîne une diminution du temps de séjour des particules fines (TCpp) non excrétées avant la première phase de cæcotrophie, mais sans variations importantes du temps de séjour des grosses particules $(>0,3 \mathrm{~mm})$. La nature des lignines ne semble pas non plus modifier les valeurs de ces 2 index.

\section{CONCLUSION}

Nos modèles alimentaires, basés sur une variation des apports en lignines sans variations des teneurs en cellulose ou en hémicelluloses, ont permis de mettre en évidence un effet négatif linéaire du taux de lignines (entre 2 et $8 \%$ ) sur la digestibilité des aliments. La digestion des constituants pariétaux est plus particulièrement affectée, alors que celle des protéines n'est pas modifiée. La décroissance de la digestibilité fécale s'accompagne d'une réduction du temps de séjour des aliments.

Les variations de digestibilité et de transit sont mieux décrites par le critère lignine $\mathrm{ADL}$ que par la lignine permanganate. La signification nutritionnelle du critère $A D L$ sera précisée dans la seconde partie de cette étude portant sur les performances de croissance et de mortalité enregistrées sur d'importants effectifs d'animaux.

\section{REMERCIEMENTS}

Ce travail a été réalisé sous la responsabilité de I'INRA, avec le soutien financier du Fonds SYPRAM. Le SYPRAM a été créé en 1990 avec des fonds apportés par les adhérents de l'AMEB (Association pour le maintien de l'élevage en Bretagne), du SNIA (Syndicat national des industriels de la nutrition animale) et du SYNCOPAC (Fédération nationale des coopératives de production et d'alimentation animales), en vue de faciliter toute action d'intérêt collectif au profit des entreprises du secteur de l'alimentation animale.

\section{RÉFÉRENCES}

Björnhag G (1972) Separation and delay of contents in the rabbit colon. Swed J Agric Res 2, 125-136

Colin M, Lebas F (1976) Méthode d'étude de la digestibilité des aliments chez le lapin. 2. Périodicité des collectes. Sci Tech Anim labo 1, 129-133

Faichney GJ (1975) The use of markers to partition digestion within the gastro-intestinal tract of ruminants. In : Digestion and metabolism in the ruminant (IW Mc Donald, ACI Warner, eds), 227-241

Falcao e Cunha $L$ (1988) Os constiutentes do parede celular no processo digestivo de coelho. Thèse docteur agronomie, Inst Sup agronomia, Univ Tecnica Lisboa, $359 \mathrm{p}$

Falcao e Cunha L, Lebas F (1986) Influence chez fe lapin adulte de l'origine et du taux de lignine altmentaire sur la digestibilité de la ration et l'importance de la cæcotrophie. $4^{e}$ Journ Rech Cunicole $\mathrm{Fr}$, Paris, 10-11 déc 1986, Communication $n^{\circ} 8,8.1$ 8.9. ITAVI ed, Paris

Fraga MJ, Pérez De Ayala P, Carabano R (1991) Effect of type of tiber on the rate of passage and on the contribution of soft feces to the nutrient intake of finishing rabbits. J Anim Sci 69, 1566-1574

Gidenne T (1986) Évolution nycthémérale des produits de la fermentation bactérienne dans le tube digestif du lapin en croissance. Relations avec la teneur en lignines de la ration. Ann Zootech 35, 121-136 
Gidenne T (1987a) Influence de la teneur en lignines des aliments sur la composition des digesta et la production de cæcotrophes chez le lapereau. Ann Zootech 36, 85-90

Gidenne T (1987b) Utilisation digestive de rations riches en lignines chez le lapin en croissance : mesures de flux et de transit dans différents segments digestifs. Ann Zootech 36, 95-108

Gidenne T (1993) Measurement of the rate of passage in restricted-fed rabbits: effect of dietary cell wall level on the transit of fibre particles of different sizes. Anim Feed Sci Technol 42, 151-163

Gidenne T, Scalabrini F, Marchais C (1991) Adaptation digestive du lapin à la teneur en consituants pariétaux du régime. Ann Zootech 40, 73-84

Giger $S$ (1985) Revue sur les méthodes de dosage de la lignine utilisées en afimentation animale. Ann Zootech 34, 85-122

Jilge $B$ (1982) Rate of movement of marker particles in the digestive tract of the rabbit. $L a b$ Anim 16, 7 11

Jung HG, Fahey GC (1983) Nutritionnal implications of phenolic monomers and lignin : a review. J Anim Sci 57, 206-219

Lenz FJ, Schurch A (1967) Uber das Verhalten von lignin im Verdauungstrakt des Kaninchens. $Z$ Tierphysiol Tierernähr FüttermittelK 22, 236-241

Merino JM, Carabano R (1992) Effect of type of fibre on ileal and fecal digestibility. J App/ Rabbit Res 15, 931-937

Monties B (1980) Les lignines. In : Les polymères végétaux (B Monties, ed), Gauthier-Villars, 122-155
Motta W (1990) Effectos de la sustitucion partial de heno de alfalfa per orujo de uva o pulpa de remolacha, sobre la utilizacion de la dieta y los rendimientos en conejos en crecimiento. Tesis doctoral, Univ Politec Madrid, $251 \mathrm{p}$

Parigi-Bini R, Chiericato $F(1980)$ Utilisation of grape marc by growing rabbits. Proc 2d Congress of the World Rabbit Science Association (WRSA ed), 15-18 april 1980, Barcelone, vol 2, 204-213

Perez JM (1991) Intérêt et limites des modèles de prévision de la valeur énergétique des aliments destinés au porc. Thèse de doctorat, université de Montpellier II, $196 \mathrm{p}$

Perez JM, Gidenne T, Lebas F, Caudron I, Arveux $P$, Bourdilion A, Duperray J, Messager B (1994) Apports de lignines et alimentation du lapin en croissance. 2. Conséquences sur les performances et la mortalité. Ann Zootech 43, 323-332

Pérez de Ayala PM (1989) Utilizacion de distintos tipos de fibra por los conejos en cebo. Tesis doctoral, Univ Politec Madrid, ETS Ing Agron, $115 \mathrm{p}$

SAS (1988) SAS/STAT Guide for personal computers (release 6.03) SAS Institute Inc, Cary, NC, $1028 \mathrm{p}$

Uden P, Van Soest PJ (1982) The determination of digesta particle size in some herbivores. Anim Feed Sci Technol 7, 35-44

Van Soest PJ, Wine RH (1968) Determination of lignin and cellulose in acid detergent fibre with permanganate. J Ass Off Anal Chem 51, 780-785

Van Soest PJ, Robertson JB, Lewis BA (1991) Methods for dietary fiber, neutral detergent fiber, and nonstarch polysaccharides in relation to animal nutrition. J Dairy Sci 74, 3583-3597 